\title{
An Analysis of Inlet Inclination on the Performance of a Gas-Liquid Cylindrical Cyclonic Separator
}

\author{
Sunday Kanshio \\ Department of Petroleum and Gas Engineering, Baze University, Abuja, Nigeria \\ Email address: \\ sunday.kanshio@bazeuniversity.edu.ng

\section{To cite this article:} \\ Sunday Kanshio. An Analysis of Inlet Inclination on the Performance of a Gas-Liquid Cylindrical Cyclonic Separator. International Journal \\ of Oil, Gas and Coal Engineering. Vol. 7, No. 3, 2019, pp. 82-88. doi: 10.11648/j.ogce.20190703.12
}

Received: August 5, 2019; Accepted: August 24, 2019; Published: September 10, 2019

\begin{abstract}
Gas-Liquid cylindrical cyclonic separator plays a significant role with regards to gas-liquid separation in the oil fields. Its major operational challenges include liquid carryover and excessive pressure losses. The Inlet geometry of a gasliquid cylindrical cyclone is one of its critical parts that affect the performance of the separator. The inlet geometry drives the performance of the separator in terms of liquid carryover and pressure drop. Traditionally, the $27^{\circ}$ downward inclined inlet is usually used because of its advantage in terms of liquid carryover operating envelope. However, detail comparison in terms of pressure drop in the gas leg of the separator is yet to be reported. In this paper, the author presents experimental results on the effect of inlet inclination on the performance of a gas-liquid cyclonic separator in terms of separation efficiency (liquid carryover) and pressure drop. The results showed that under the same inlet conditions, the liquid carryover operating envelope of the $27^{\circ}$ downward inclined inlet pipe cyclonic gas-liquid separator is slightly wider than the horizontal inlet of the same separator. However, the pressure drop across the gas leg of the $27^{\circ}$ downward inclined inlet pipe cyclonic gas-liquid separator is far greater than the horizontal inlet of the same separator. The paper concludes that, where there is a strict requirement on pressure drop, the horizontal inlet cyclonic separator should be favoured against the $27^{\circ}$ inclined inlet.
\end{abstract}

Keywords: Multiphase Separation, Cyclonic Separator, Tangential Inlet, Inlet Inclination, Liquid Carryover, Inlet Nozzle, Pressure Transducer

\section{Introduction}

Usually, the hydrocarbon fluids from the wellhead is a multiphase mixture of water, oil and gas. The mixture must be separated into its individual components to produce market products, namely, oil and natural gas. Separators are the process equipment used in the oil and gas fields to accomplish the task of separating the multiphase mixture into crude oil and natural gas. Traditionally, gravity separators are usually used in separating the multiphase produced fluids into their respective components, namely, oil, water and gas. In the offshore sector of the oil and gas industry because of space and weight limitation, compact separators such as the gas-liquid cylindrical cyclone (GLCC) are more attractive. The GLCC is cheap, pipe rated, light weight, compact and simple [1]. The operating principle of the GLCC separator is based on the combine effects of centrifugal and gravity forces. The centrifugal force is created because of the tangential inlet of the separator, which causes the multiphase flow to spin on entering the separator. Due to centrifugal force, the time required for phase separation is reduced and therefore, the size of the separator. In the oil and gas industry, the application of this type of separator is attractive, especially where space is a constraint such as offshore platforms, subsea and downhole. Another application of GLCC separator is as knock-out vessel for removal of liquid droplets at the inlet of a gas turbine. The concept of GLCC was developed by Chevron at the University of Tulsa [2]. Since then, tons of experiment has been conducted on the GLCC with the recent work being conducted by Kolla et al., [3-5].

Liquid carryover (LCO) and pressure drop are some of the performance indicators of any gas-liquid cyclone separator [6]. The LCO could be defined as the fraction of liquid that is transported out of the separator by the gas stream. While the LCO generally pertains to separation efficiency, pressure drop, on the other hand, pertains to the operating cost of the equipment. The ranges of gas and liquid flow rates within 
which the separator most be operated to achieve an efficient separation are usually represented graphically (Figure 1) and referred to as the operating envelope of the separator [7-10]. The critical operating line on the operating envelope is the boundary between partial and complete gas-liquid separation. This means that the separator must be operator below the critical separation line for a complete gas-liquid separation to be achieved.

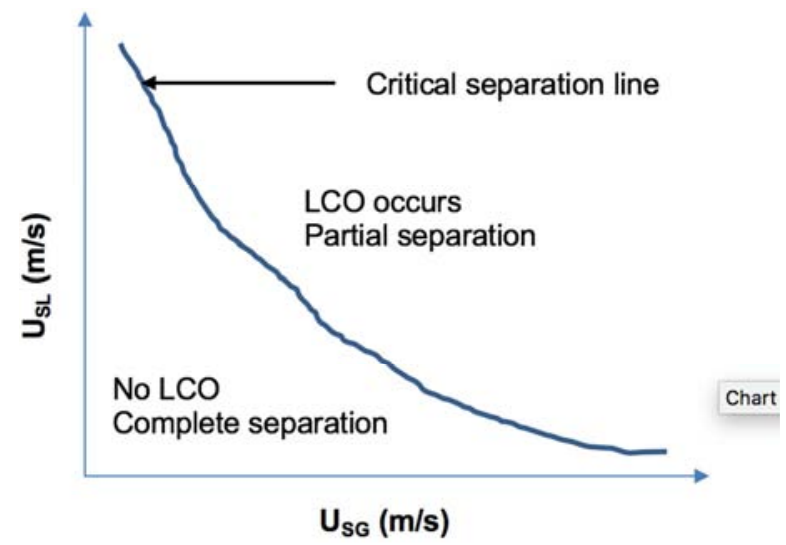

Figure 1. The shape of liquid carryover operating envelope for gas-liquid cylindrical cyclonic separator.

The operating envelope for liquid carryover determines the gas and liquid handling capacity of any gas-liquid cyclone separator. The narrow operating envelope for liquid carryover and pressure drop across the gas leg of the separator has always been the major operational challenge of the GLCC separator. The GLCC inlet pipe angle and size were found by researchers at the University of Tulsa to have an effect on the LCO and hence, the separation efficiency of the separator [912]. Kouba [2] investigated the effect of inlet inclination on the separation efficiency of gas-liquid cylindrical cyclone (GLCC) separator. The diameter of the GLCC separator inlet pipe used was the same as the separator diameter (i.e. 3 inches). However, the inlet diameter was reduced using a slot-wall nozzle. A horizontal and $27^{\circ}$ downward inclined inlet pipe were compared experimentally. It was concluded that the $27^{\circ}$ downward inclined inlet pipe gave a better separation efficiency compared to the horizontal inlet. The reasons given for the increase in separation efficiency include pre-separation at the inlet due to stratified flow and fluid entry below the separator inlet axis. However, the effect of the $27^{\circ}$ downward inclined inlet pipe on the pressure drop across the separator was not studied.

Most of the subsequent pieces of published work on GLCC separator adopted Kouba's idea of using an inclined tangential inlet pipe with the same size as the GLCC separator $[9,10,13,14]$. To improve the separation performance of GLCC, various nozzle designs have been studied [15-17]. The concluding remark from the existing studies is that nozzle increased the tangential velocity and hence, separation efficiency. More ever, the effectiveness of the nozzle in terms of separation enhancement depends on its shape and size. However, the effect of those nozzles on the pressure drop across the gas leg of the separator was not presented.

Most of the existing studies on liquid carryover in GLCC separator were based on visual observation of liquid droplets in the gas outlet. In an attempt to estimate the percentage of liquid carryover in a 3 inch diameter gas-liquid cylindrical cyclone (GLCC) separator, Chirinos et al., [9] installed a 6 inch diameter liquid-trap-pipe in the outlet of the GLCC separator so as to capture the liquid leaving the separator. Rosa et al.[18] adopted a similar approach by installing a demister with a calibrated chamber in the outlet of their cyclonic separator (CS) to capture any liquid in the gas and measure the LCO. Hreiz et al., [19] investigated the effect of inlet nozzle on the performance of GLCC and concluded that flow restriction by using an inlet nozzle increases the centrifugal force and enhances the separation efficiency. Hreiz, like other researchers, did not consider the effect of inlet geometry on the pressure drop across the gas leg of the GLCC separator.

There are scarce experimental studies regarding the effect of separator inlet configuration on the pressure drop across GLCC separator. Generally, the pressure drop across GLCC separator have been measured for reasons other than the determination of inlet geometry effects. Kouba et al., [2] measured the pressure drop across GLCC separator to estimate liquid holdup under zero-net liquid flow condition. Kristiansen et al., [20] measured the pressure drop across GLCC separator only for the purpose of obtaining pressure drop as the operating parameter rather than a performance parameter. There is a need to evaluate the effect of inlet geometry on the pressure drop across the gas leg of the separator to decide the best inlet design.

In this work, a wire mesh sensor and pressure transducer were used to measure the liquid holdup and pressure drop, respectively in the gas disengagement section of the separator. The data obtained were analysed quantitatively to discriminate between critical and liquid carryover conditions. The major contribution of this paper is the presentation of experimental data of pressure drop and LCO operating envelop for various inlet geometry. Also, another contribution is the comparison between the traditional $27^{\circ}$ inclined inlet and the horizontal inlet in terms of the LCO and pressure drop.

\section{Experimental Setup and Procedure}

Description of the test facility: The gas-liquid pipe cyclone (GLPC) separator was fabricated and installed in a test facility is at Cranfield University, UK. Similar to other cyclonic separators, the GLPC has a tangential inlet with a diameter which is half that of the separator. The operating principle of the GLPC is the same as that of the gas-liquid cylindrical cyclone (GLCC) separator. The difference between the two is the inlet pipe geometry. While the GLCC has an inclined inlet pipe with a diameter the same as the separator, the GLPC has a horizontal inlet pipe whose diameter is half the diameter of the separator. 

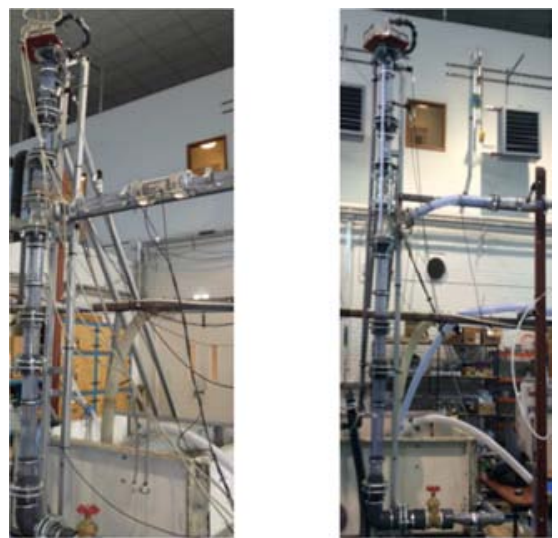

Figure 2. Photo of the gas-liquid pipe cyclonic separator (GLPC): Left is horizontal inlet and right is $27^{\circ}$ downward inclined inlet.
As shown in Figure 2, Figure 3 and Figure 4, the test facility is a closed loop system consisting of the fluids supply and metering section; GLPC separator, and fluids return section. Air and water were used as test fluids and referred to as gas and liquid, respectively in this work. The gas (air) was supplied by a compressor and metered using Endress+Hauser thermal mass flow meter (Proline t-mass 65). Water was supplied to the flow loop by Certikin Aquaspeed self-priming pump which has a maximum duty of $41 / \mathrm{s}$ at $3 \mathrm{barg}$. It was metered using $\mathrm{ABB}$ electromagnetic flow meter. The test section consists of the GLPC separator, where the separation of gas and liquid takes place. Two types of inlet configuration, as shown in Figure 2, were investigated. The separator dimension is as shown in Table 1.
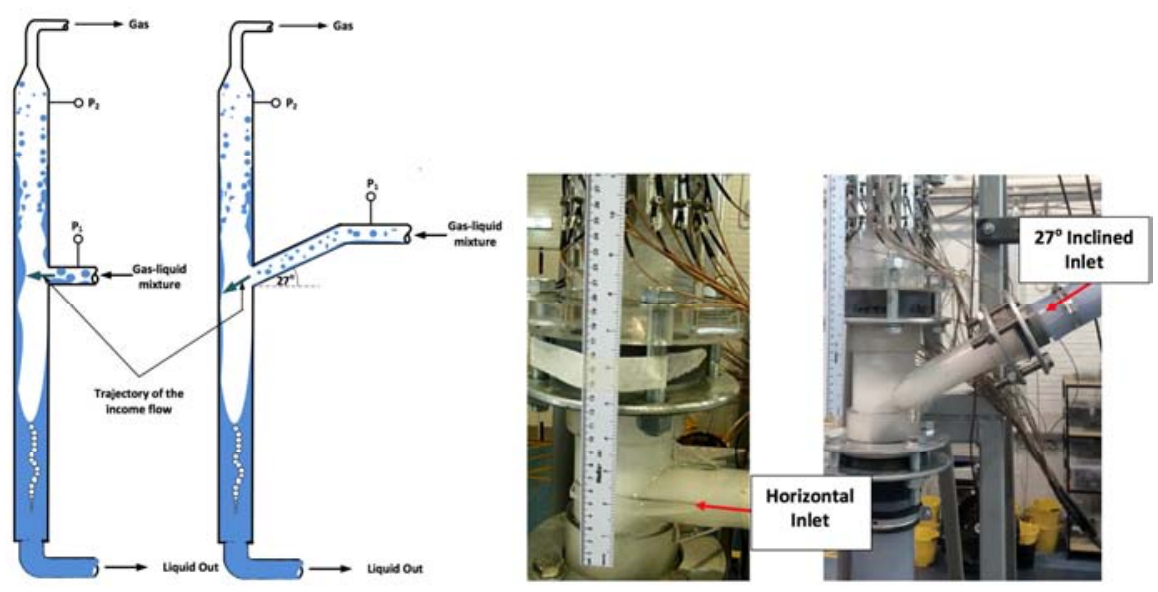

Figure 3. Horizontal (Left) and the inclined (right) tangential inlet of GLPC separator.

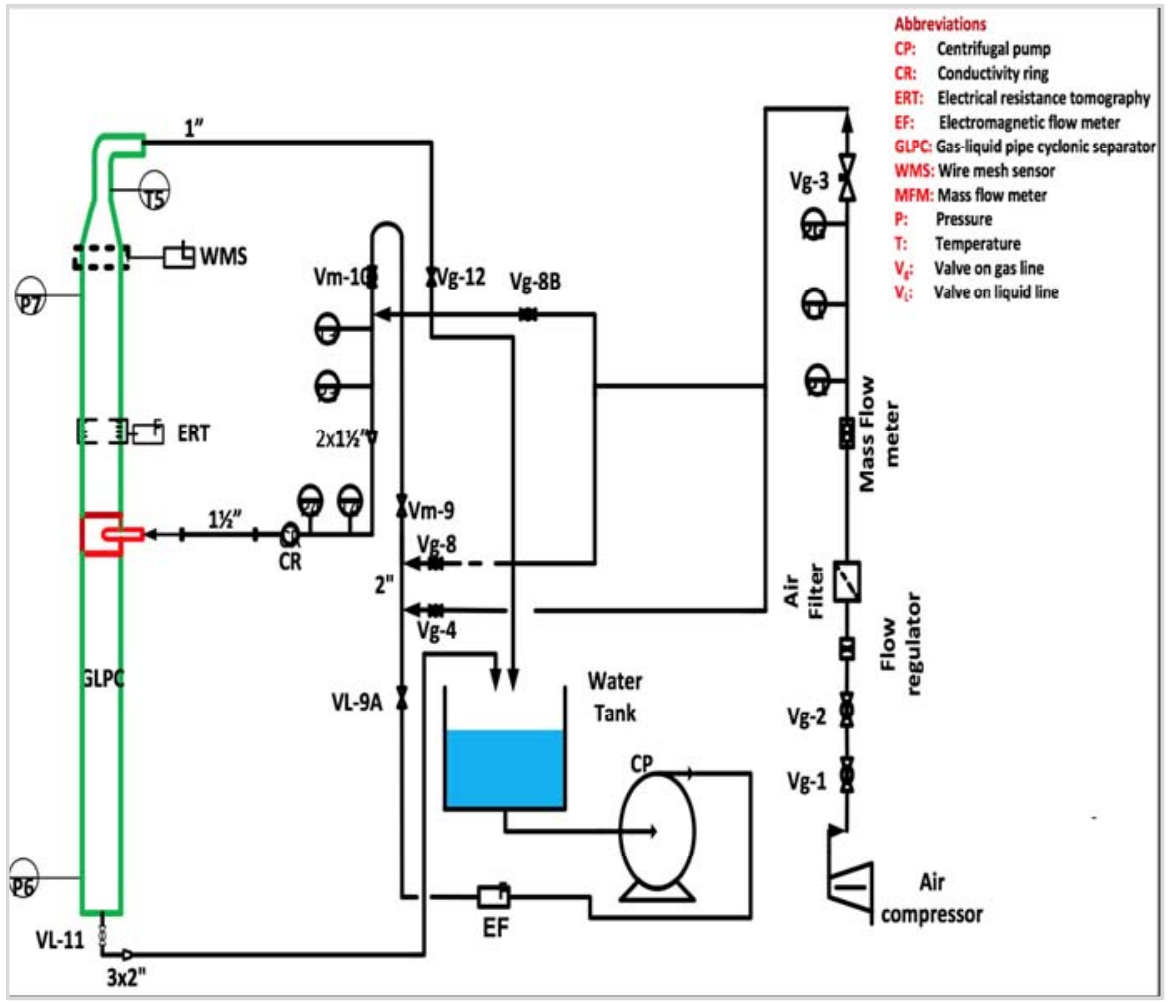

Figure 4. Schematic of the experimental setup. 
Table 1. Dimensions of the GLPC separator.

\begin{tabular}{ll}
\hline Parts & Dimension $(\mathbf{m m})$ \\
\hline Separator diameter & 76.10 \\
Inlet pipe diameter & 38.10 \\
Gas outlet diameter & 25.40 \\
Liquid outlet diameter & 50.80 \\
Height above inlet & 1300 \\
Height below inlet & 1600 \\
\hline
\end{tabular}

Separator inlet geometries: The inlet geometries tested include $27^{\circ}$ inclined inlet, horizontal inlet and nozzles. The separator inlet nozzle was varied to investigate their effect on liquid carryover and pressure drop. These nozzles were

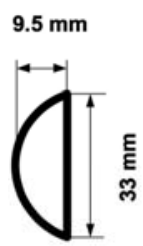

D-Noz-1

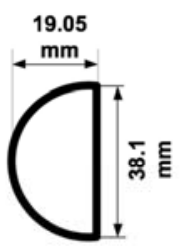

D-Noz-2 carefully installed in the inlet pipe and were aligned with the wall of the separator to give a D-shape entrance. Two basic principles underpinned the inlet nozzle design: increase inlet fluid velocity and maximise the fluid rotation on the separator wall. The nozzles have two sides: flat and curved side. The flat side has a long-tapered end to convey the fluid into the reduced flow area gently. Since these three inlet nozzles have the shape of D-shape, they are being referred to respectively as D-Noz-1 and D-Noz-2. The schematic drawing of the inlet nozzles opening, and the isometric view is presented in Figure 5.

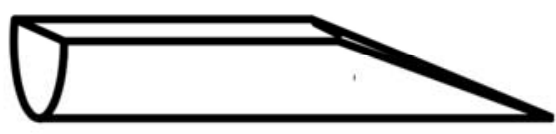

Isometric View of the insert

Figure 5. GLPC separator inlet nozzle.

Instrumentation and data acquisition system: The instruments used for this research include pressure transducer, temperature probe and electrical resistance tomography (ERT). The ERT was used for online flow imaging to view the structure of the flow near the inlet. The ERT is a non-intrusive measurement technique by which information about the electrical properties of fluids in a process volume is inferred from the periphery electrodes measurement. The ERT used in this study consist of a dualplane sensor each having 16 stainless steel electrodes mounted on the periphery of the GLPC separator. A data acquisition system (DAS) developed by ITS Plc, Manchester, UK, was used to acquire data from the sensor. The data acquisition and transfer speed of the system was approximately 1000 frames per second. The sensitive coefficient back projection algorithm was used for image reconstruction. Pressure measurement forms a critical element of this research. The operating pressure of the separator and flowline pressure was measured using pressure transducer manufacture by Drunk GE. In the upstream fluid supply section, two pressure transducers were installed: one for measuring single-phase air pressure and the other for measuring the pressure of the two-phase mixture at the separator inlet. In the separator, two pressure transducers were installed in the gas disengagement section of the separator. These pressure transducers were all absolute pressure transducer with rangeability between 0-6 bar and uncertainty of $\pm 0.15 \%$ of full scale.

\section{Results and Discussion}

The results and discussions are divided into two sections: LCO performance and pressure drop in the gas leg section of the separator for both $27^{\circ}$ inclined inlet and horizontal inlet, respectively. In section 3.1 , the performance in terms of the operating envelope for liquid carryover for both the horizontal and inclined inlet with and without an inlet nozzle. In section 3.2, data for separator performance in terms of pressure drop is presented and analysis for all the inlet geometry.

\subsection{Liquid Carry over (LCO) Performance}

The liquid carryover, which is considered in this work as an indication of the separation efficiency, is presented on the operating envelope curve. The operating envelope for LCO was determined by fixing the liquid flow rate and increasing gas flow gradually until droplets start jumping towards the outlet. This was followed by observing the gas outlet to ensure that there were no drops. This was confirmed by monitoring pressure signals and visualising WMS dynamic image display to ensure that there were little signals fluctuations before the data was acquired. During liquid carryover, there were significant fluctuations of pressure and WMS images. The operating envelope is presented for both the horizontal and inclined inlet.

\subsubsection{Full Pipe Bore (No Nozzle) Inlet}

Two tangential inlets geometry, namely, horizontal and $27^{\circ}$ downward inclined, as shown in Figure 3, were investigated. For the full pipe bore test, the flow at the inlet of both horizontal and inclined inlet was not restricted with a nozzle. The operating envelope for liquid carryover of the two tangential inlets is plotted, as shown in Figure 6. The superficial velocity used in plotting the operating envelope was calculated from the full pipe bore, which is $38.1 \mathrm{~mm}$ ID. Using the horizontal tangential inlet as the base case, an improvement in separation efficiency was achieved with the downward inclined tangential inlet. However, this improvement in separation efficiency was marginal. These 
results are contrary to what was reported by Kouba et al. (1995). The reason for this marginal improvement in separation performance is attributed to the use of an inlet pipe diameter $(38.1 \mathrm{~mm}$ ID) that is half of the separator diameter (76.2mm ID). This inlet pipe diameter is not large enough to cause stratified flow in the inclined inlet within the flow rates investigated. The only advantage of the inclined separator inlet in this study is the fact that the liquid jet was introduced below the inlet centreline of the separator. The reason for inclining the GLCC inlet is to cause stratify flow and promote pre-separation of liquid from gas just before the inlet nozzle. All the data points on the operating envelope in the present study shown were far beyond the threshold for stratified flow. This suggests that there is no need for downward inclined tangential inlet when the inlet diameter is half the separator diameter.

In the case of GLCC, it was reported that a nozzle was used to reduce entrance by $25 \%$ of the inlet pipe cross sectional area (Chirinos et al., 2000). This implied that stratified flow is possible, and swirl could be generated because of the increase in velocity at the nozzle. Based on the observed difference, it is pertinent to assert that separation efficiency is not only the function of inlet pipe angle inclination. One must consider the ratio between the inlet pipe and separator diameter as well as the effect of flow development in the inlet pipe. The flow development depends on the length of straight pipe before the nozzle. In the present study, the straight pipe before the inlet section was $3.5 \mathrm{~m}$ long. The inclined inlet section is about $0.7 \mathrm{~m}$ long. The $3.5 \mathrm{~m}$ straight pipe brought about flow development that positively helped the separation efficiency for both horizontal and inclined inlet.

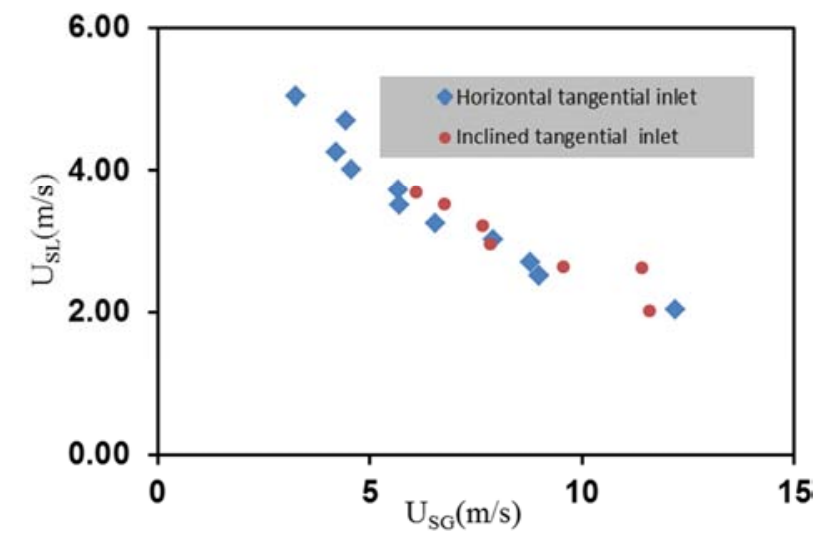

Figure 6. Operating envelope for LCO of horizontal and downward inclined tangential inlet $76.10 \mathrm{mmID}$ GLPC separator.

\subsubsection{Inlet Pipe with a Nozzle}

In designing a gas-liquid pipe cyclone, the size and shape of the nozzle need to be carefully selected as this has a direct influence on swirl intensity.

In this work, a nozzle with D-shape opening, as explained in Section 2 was inserted into the horizontal and inclined tangential inlet respectively to reduce the cross-sectional area of their bore. Using the full pipe bore of the horizontal inlet as the base case, the D-Noz-1 improves the operating envelope for LCO of both horizontal and inclined inlet separator, as shown in Figure 7. This is because the nozzle increases the tangential velocity and produces a stable film layer on the separator wall. However, the improvement in the operating envelope for LCO compare to the full pipe bore was marginal. The present results on separation improvement by using a nozzle agreed with Hreiz et al., (2014) and Uvwo [17] findings, although the nozzles used in this work are of different geometry. Interestingly, the D-Noz-2 shows a negative effect on the operating envelope for LCO. This is because the nozzle over restricted the flow area, thereby causing large shear effects on the liquid, which consequently creates liquid droplets which were easily lifted by the gas stream out of the separator. When the D-Noz-2 was inserted into the inclined inlet, the flow restriction far exceeded that of the horizontal inlet, in fact, high liquid and gas flow into the separator was not possible due to large backpressure.

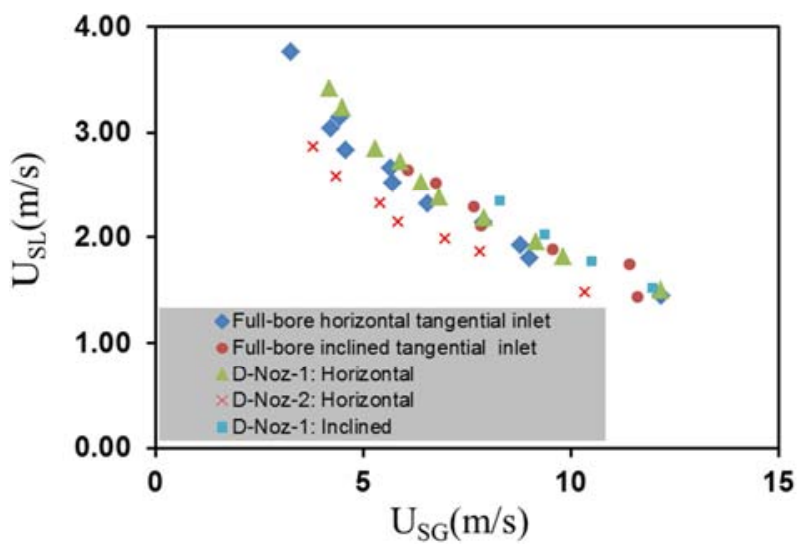

Figure 7. Nozzle effect on operating envelope for LCO.

\subsection{Pressure Drop Performance}

Pressure drop results for horizontal and inclined full pipe bore inlet and nozzle are presented in this section.

\subsubsection{Full Inlet Pipe Bore}

The pressure at the inlet pipe, $\mathrm{P}_{1}$, and the separator top, $\mathrm{P}_{2}$, were measured using absolute pressure transducer, respectively. The pressure measurement positions are shown in the schematic diagram in Figure 3. The pressure drop data set is for separator operating conditions before the occurrence of LCO. The pressure drop was calculated using equation 1.

$$
D p=P_{1}-P_{2}
$$

The pressure drop as a function of superficial gas velocity $\left(\mathrm{U}_{\mathrm{sg}}\right)$ at constant superficial liquid velocity is presented in Figure 8. As expected, pressure drop for both horizontal and inclined inlet increased with increasing superficial gas velocity. This is because of the combined effect of entrance losses and losses due to friction, both, which eventually increases as the gas velocity increases. However, an interesting result is seen when the pressure drop for the horizontal inlet is compared against the inclined inlet. The inclined inlet gives the largest pressure drop; ranging from $3 \%$ to $15 \%$ of inlet pressure from low to high superficial gas 
velocity. When compared with the horizontal inlet, a difference $13 \%$ to $138 \%$ from low to high superficial gas velocity can be seen. These wide differences in pressure drop for the inclined inlet may sound strange but looking the trajectory (as sketched in Figure 3) of the fluids at the entrance of the separator it is not surprising. In the case of the horizontal inlet, the fluid followed the curve wall smoothly on entering the separator because there is no change in flow direction and there is no impingement on the separator wall hence energy loss is minimal. In the case of the inclined inlet, there is a sudden change in flow direction from a horizontal pipe to incline; therefore, the fluid impinges on the wall before taking the first turn on the separator wall. It is believed that this impingement brings additional dissipation of kinetic energy that resulted in an increase in pressure drop. Experimental observation revealed that the swirl generated by the inclined inlet was less intense compare to the horizontal inlet because the impingement reduces the intensity of the swirl motion.

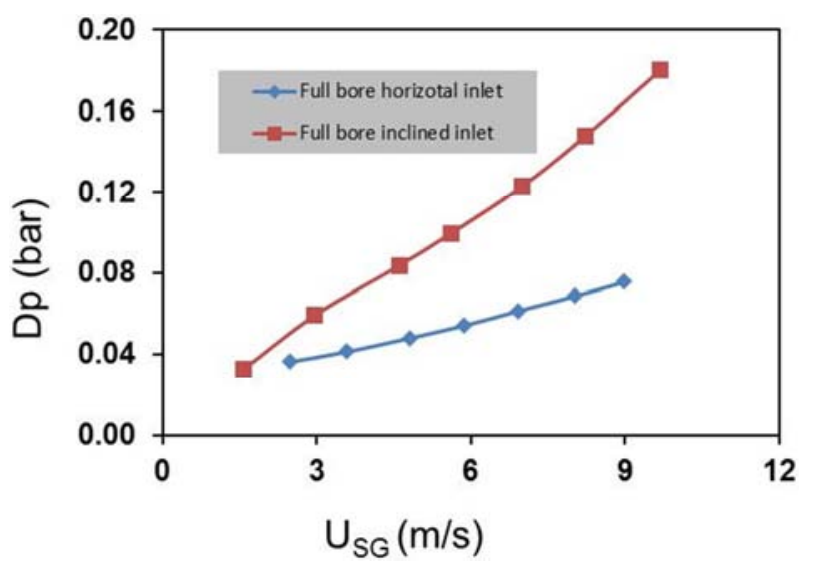

Figure 8. Pressure drop against superficial gas velocity at fixed superficial liquid velocity $\left(U_{S L}=1.8 \mathrm{~m} / \mathrm{s}\right)$ for horizontal and inclined tangential inlet respectively.

\subsubsection{Inlet Pipe with a Nozzle}

The effect of the nozzle on pressure drop in cyclonic pipe separator is just as important as operating envelope for LCO. As shown in Figure 9, the pressure drop for each data point corresponds to the data points on the operating envelope (Figure 6). The pressure drop of the full-bore horizontal inlet is used as the base case. The pressure drop for all the nozzles generally showed an increasing trend in the direction of increasing superficial liquid velocity. This is because, at high superficial liquid velocity, the average cross section liquid holdup in the inlet pipe is also high resulting in high mixture density. Therefore, the pressure drop due to nozzle effect would increase according to Equation 2. Furthermore, increasing inlet, liquid flow rate also increases the liquid holdup at the inlet and consequently increases the liquid holdup in the separator. Increasing liquid holdup results in a corresponding increase in interfacial friction and therefore increase in pressure drop.

$$
\mathrm{dp}_{\mathrm{noz}}=0.5 \rho v^{2}
$$

Where $d p_{n o z}, \rho$ and $v$ represent pressure drop due to the nozzle, density and velocity respectively.

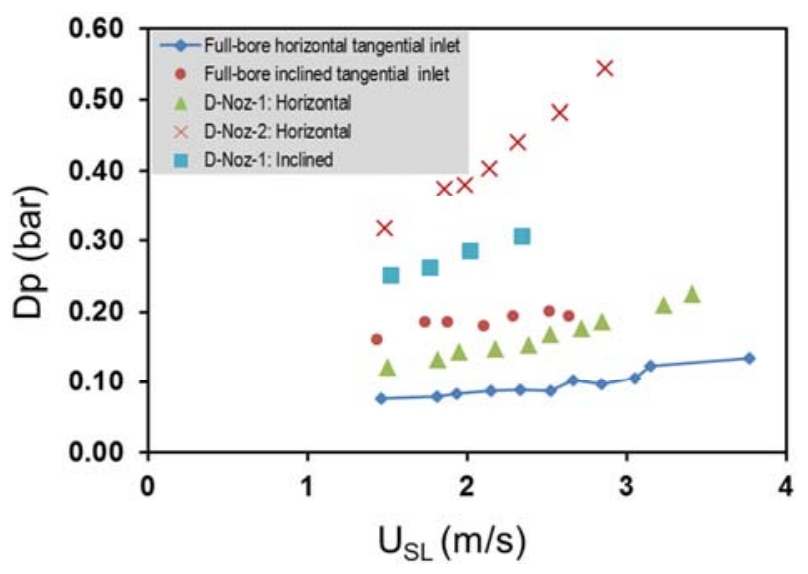

Figure 9. Effect of the nozzle on pressure drop: the superficial liquid velocity was calculated based on the full pipe bore area $(d=38.10 \mathrm{mmID})$.

The D-Noz-2 caused the greatest pressure drop. This is not surprising because of the large backpressure that the nozzle generated because of large flow restriction at the separator entrance. Considering that D-Noz-2 also produces the highest velocity, it is justifiable according to Equation 2 that the pressure drop is high compare to another nozzle, especially the base case. The pressure drop of the inclined inlet D-Noz1 far exceeds that of the horizontal inlet D-Noz-1 for the same reason as suggested in for the full-bore inclined inlet in Section 3.2.1 above.

\section{Conclusion}

The performances of GLPC separator with respect to operating envelope for LCO and pressure drop has been analysed. The full pipe bore inclined tangential inlet gives a marginal improvement on the operating envelope for LCO as compared to the horizontal type but brought a significant increase in pressure drop. The D-Noz-1 nozzle gave a marginal improvement on operating envelope for LCO for both inclined and horizontal inlet but brought a considerable increase in pressure drop. However, D-Noz-2 nozzle performs poorly in terms of pressure drop and operating envelope for LCO. From the experimental study, it could be concluded that where the inlet diameter is half the separator diameter, there is no advantage in using an inclined $27^{\circ}$ inlet pipe.

\section{References}

[1] Msi, "A State-of-the-art, Highly Efficient and Reliable Compact Separator Providing Optimum Gas-Liquid Separation Performance at Minimum cost," 2006. [Online]. Available: http://msieng.com/pages/sub/other/glcc.htm.

[2] G. E. Kouba, O. Shoham, and S. Shirazi, "Design and performance of gas-liquid cylindrical cyclone separators," in Proceedings of the BHR Group 7th International Meeting on Multiphase Flow., Cannes, France, 1995, pp. 307-327. 
[3] S. S. Kolla, R. S. Mohan, and O. Shoham, "A Study on the Effect of Fluid Properties and Watercut on Liquid Carry-Over in Gas-Liquid Cylindrical Cyclone Compact Separators," J. Fluids Eng., vol. 141, no. 9, pp. 91303-91310, Apr. 2019.

[4] S. S. Kolla, R. S. Mohan, and O. Shoham, "Effect of Liquid Level on Gas Carry-Under in GLCC Compact Separators," no. 51555. p. V001T07A005, 2018.

[5] S. S. Kolla, R. S. Mohan, and O. Shoham, "Computational Fluid Dynamics Study on the Effect of Inlet Modifications of Gas-Liquid Cylindrical Cyclone (GLCCO) Compact Separators," no. 58066. p. V01CT14A007, 2017.

[6] V. van A. N. Nanninga, J. W. F. Janssen, "Gasunie/CDS Improvement of the Gasunie Cyclone Gas-Liquid Separator," 2001.

[7] Z. Atakan, Y. D. Chin, P. P. Lang, and S. Iyer, "Design and Operability Considerations of the Gas Flowline at Parque das Conchas (BC-10) Ostra Field," in Offshore Technology Conference, 3-6 May, Houston, Texas, USA, 2010.

[8] T. Yue et al., "Experimental and numerical study of Upper Swirling Liquid Film (USLF) among Gas-Liquid Cylindrical Cyclones (GLCC)," Chem. Eng. J., vol. 358, pp. 806-820, 2019.

[9] W. A. Chirinos, L. E. Gomez, S. Wang, R. S. Mohan, O. Shoham, and G. E. Kouba, "Liquid Carry-Over in Gas/Liquid Cylindrical Cyclone Compact Separators," SPE J., vol. 5, no. 3, pp. 259-267, Jan. 2000.

[10] O. Shoham and G. E. Kouba, "State of the Art of Gas/Liquid Cylindrical-Cyclone Compact-Separator Technology," J. Pet. Technol., vol. 50, no. 7, pp. 58-65, Jan. 1998.

[11] G. E. Kouba, S. Wang, L. E. Gomez, R. S. Mohan, and O. Shoham, "Review of the State-of-the-Art Gas/Liquid Cylindrical Cyclone (GLCC) TechnologyField Applications," International Oil \& Gas Conference and Exhibition in China. Society of Petroleum Engineers, Beijing, China, 2006.
[12] I. Arpandi, A. R. Joshi, O. Shoham, S. Shirazi, and G. E. Kouba, "Hydrodynamics of Two-Phase Flow in Gas-Liquid Cylindrical Cyclone Separators," in SPE Annual Technical Conference \& Exhibition held in Dallas, U.S.A., 22-25 October 1995., 1996.

[13] S. Movafaghian, J. a Jaua-marturet, R. S. Mohan, and O. Shoham, "The effects of geometry, fluid properties and pressure on the hydrodynamics of gas-liquid cylindrical cyclone separators," vol. 26, pp. 999-1018, 2000.

[14] R. Hreiz, C. Gentric, N. Midoux, R. Lainé, and D. Fünfschilling, "Hydrodynamics and velocity measurements in gas-liquid swirling flows in cylindrical cyclones," Chem. Eng. Res. Des., vol. 92, no. 11, pp. 2231-2246, 2014.

[15] R. Hreiz, R. Lainé, J. Wu, C. Lemaitre, C. Gentric, and D. Fünfschilling, "On the effect of the nozzle design on the performances of gas-liquid cylindrical cyclone separators," Int. J. Multiph. Flow, vol. 58, pp. 15-26, Jan. 2014.

[16] N. Barbuceanu, S. Scott, A. Texas, and S. Scott, "SPE 71555 Novel Inlet Design Expense Range of Operability for Compact Separator," Spe 71555, 2001.

[17] I. Uvwo, "Expanding the Operational Envelope of Compact Cylindrical Cyclone Gas / Liquid Separators Using a Variable Inlet-Slot Configuration Expanding the Operational Envelope of Compact Cylindrical Cyclone Gas / Liquid Separators Using a Variable Inlet-Slot Confi," no. December 2004.

[18] E. S. Rosa, F. A. França, and G. S. Ribeiro, "The cyclone gasliquid separator: operation and mechanistic modeling," J. Pet. Sci. Eng., vol. 32, no. 2, pp. 87-101, 2001.

[19] R. Hreiz, R. Lainé, J. Wu, C. Lemaitre, C. Gentric, and D. Fünfschilling, "On the effect of the nozzle design on the performances of gas-liquid cylindrical cyclone separators," Int. J. Multiph. Flow, vol. 58, pp. 15-26, Jan. 2014.

[20] O. Kristiansen, Ø. Sørensen, and O. R. Nilssen, "CompactSep TM - Compact Subsea Gas-Liquid Separator for HighPressure," 2016. 